\title{
Kinematical Analysis on the Last Two Steps and Take-off Technique of Chinese Elite Male long Jumpers
}

\author{
Qiong Zhou, Zu-Hua Xie, Teng-Fei Yao, Wen-Juan WU, Jia-Li Xu, Jiang-Hua Li* \\ Key Lab of Training, Monitoring and Intervention of Aquatic Sports of General Administration of Sport of China, Institute of \\ Physical Education, Jiangxi Normal University, Nanchang, China \\ lijianghua8@sina.com
}

\begin{abstract}
Long jump is composed of the technical movements such as run-up, take-off, vacating and landing. The run-up and take-off techniques are the key factors to determine the performance in a long jump, especially the last several steps. In this paper, the last two steps and takeoff technique of the three Chinese elite male long jumpers, who entered the final competition in the World Athletics Championships in 2015, were analyzed using Dartfish video analysis system. The results showed that the jumping angle of the Chinese elite male long jumpers was at a reasonable range, but compare with the most elite athletes in the world, the last two steps and the takeoff technique of the Chinese elite male athletes were not perfect. The step distances of the last two steps of the most elite athletes in the world were "large-small", while these of the Chinese elite male long jumpers were "small-large", which resulted in a loss of horizontal speed; the takeoff sector angle of the Chinese elite male long jumpers was bigger than that of the most elite athletes in the world, which led to a loss of vacating height. Therefore, the last two steps and the takeoff technique of Chinese long jumpers are still needed to be improved.
\end{abstract}

Keywords - Men's long jump; approach; the last two steps; technical analysis

\section{INTRODUCTION}

Long jump is composed of the technical movements such as run-up, take-off, vacating and landing. The run-up and takeoff techniques are the key factors to determine the performance in a long jump, especially the last several steps. This requires athletes to have a good and comprehensive Physical qualities and accurate grasp of advanced techniques. For a long time, with the development of sports science and technology, people have carried on systematic and extensive research on long jump techniques from the aspects of sports biology, anatomy and psychology, and accumulated more research results. Resulting in long-distance technology and theory has made continuous progress and development, so that long-distance sports performance has been gradually improved. Jumping, jumping, vacating and landing and other technical movements, in recent years, domestic and foreign scholars on the run-up and take-off technology made a lot of research to prove that the run-up and take-off technology is an important technical aspects of the project and is the key factor in determining the results [1], which is mainly concentrated in the final stage of the run-up, especially the last few steps, because the last few steps will determine the run-up effect, take-off at various stages of the relevant angle to determine the take-off quality and affect the final race results. Men's long jump plays an important role in the development of competitive sports in China. At the 2015 World Athletics Championships, there are three Chinese athletes on behalf of Asians for the first time to enter the final, but there is little comparative analysis between the Chinese elite long jump athletes and the foreign excellent athletes in this competition. Therefore, the paper analyzes the technical characteristics of the last two steps and the take-off action of three Chinese athletes and three foreign athletes in order to find the gap with the excellent male long jumpers from abroad. And put forward specific recommendations.

\section{SUBJECTS AND METHODS}

\section{A. Subjects}

Six elite male long jumpers, who participated in the final competition of the World Athletics Championships held in Beijing in 2015, were analyzed using Dartfish video analysis system. As shown in table I, three of them were from China, and ranked at the $3^{\text {rd }}, 4^{\text {th }}$ and $5^{\text {th }}$ place. The 3 others were from England, Australia and Russia.

TABLE I. BASIC INFORMATION OF THE TOP 6 ATHLETES OF THE MEN'S LONG JUMP EVENT OF THE2015 WORLD ATHLETICS CHAMPIONSHIPS

\begin{tabular}{ccccc}
\hline Nationality & Name & Born & $\begin{array}{c}\text { Achieve- } \\
\text { ment }\end{array}$ & Ranking \\
\hline England & $\begin{array}{c}\text { Greg } \\
\text { RUTHERFORD }\end{array}$ & 1986.11 & 8.41 & 1 \\
Australia & Fabrice LAPIERRE & 1983.11 & 8.24 & 2 \\
China & Jianan Wang & 1996.08 & 8.18 & 3 \\
China & Xinglong GAO & 1994.03 & 8.14 & 4 \\
China & Jinzhe Li & 1989.09 & 8.10 & 5 \\
Russia & $\begin{array}{c}\text { Aleksander } \\
\text { MENKOV }\end{array}$ & 1990.12 & 8.02 & 6 \\
\hline
\end{tabular}

\section{B. Methods}

Method of documentation we've consulted a number of electronic journals and documents on the analysis of long jump 
sports at home and abroad. We are aware of the approach of long jump, the research status of take-off technique, the development trend and existing problems and at the same time deepen the understanding of the characteristics of the project, which can lay a solid foundation for this research.

Image analyses were performed using Dartfish video analysis system. The best scores of the athletes were obtained first, and then the data were analyzed.

Statistical analyses were performed using IBM SPSS Statistics software version 20.0 (SPSS Inc., Chicago, IL, USA). The data are expressed as the means \pm standard deviations (SDs) and compared using $\mathrm{T}$ tests. The significance level was set at 0.05 .

\section{RESUltS AND DiscUSSION}

\section{A The last two steps}

The task of the long jump is to get the maximum level of speed and prepare for the accurate and fast and effective takeoff technically, physically and psychologically. The last two steps of the run-up is the key to take-off jump, the pedal accuracy, Take-off angle and the vacated jump on the final results have a significant impact [2]

Step size is another important factor affecting pedal accuracy. The research of jumping class project focuses on the characteristics of the last two steps. The last step of the runnerup is closely related to the characteristics of the athletes' body function and the technical characteristics of the run-up. There are individual differences, The study shows that in the long jump, the penultimate step compared to the last step, within a certain range is shorter, the faster the pedal, the more can shorten the distance between the foot and the body center of gravity projection point, which is conducive to cut the loss of the level of the speed and make the run up and the takeoff more close for getting more sufficient and reasonable set-off and flight. But if the last step too small will affect the pedal and stretch of the takeoff leg and the acquisition of vertical speed in the taking off moment [3].

As can be seen from Table II and Fig.1 the penultimate step of the six athletes and the penultimate step of the study showed a descending trend, But the last 1 step of Chinese athletes Jinzhe Li and Aleksandr MENKOV is slightly greater than the penultimate step; the penultimate step average value of 3 foreign athletes is $2.47 \mathrm{~m}$ while our athletes is $2.33 \mathrm{~m}, 14 \mathrm{~cm}$ shorter than foreign athletes, which shows a significant difference. The last one step average value of our athletes is $2.45 \mathrm{~m}$.while the foreign athletes is $2.32 \mathrm{~m}$. The last step length of our athletes is larger than the penultimate step and is larger than that of foreign athletes; The study found that the penultimate step length of the world-class elite male long jump athletes is generally about $2.45 \mathrm{~m}$, the last step length is about $2.20 \mathrm{~m}$ [4]; In addition, seen from the decreasing range of the two steps, the penultimate step of foreign athletes is greater than the last step, the difference between the two is $15 \mathrm{~cm}$, and the average number of athletes in China reached the penultimate step is less than the last step 1, the difference between $12 \mathrm{~cm}$, the last two steps on the board "small - big" Situation, which led to the big step in the final stage and affect the pedal and stretch of the take-off leg and the acquisition of vertical speed in the taking off moment.

TABLE II. CHARACTERISTICS OF THE LAST TWO STEPS OF THE RUN - UP

\begin{tabular}{ccccc}
\hline Name & $\begin{array}{c}\text { Achieve } \\
\text {-ment }\end{array}$ & $\begin{array}{c}\text { Countdown } \\
\text { Step 2 }\end{array}$ & $\begin{array}{c}\text { Countdown } \\
\text { Step 1 }\end{array}$ & Difference \\
\hline Greg Rutherford & 8.41 & 2.61 & 2.34 & 27 \\
Fabrice Lapier & 8.24 & 2.45 & 2.27 & 18 \\
Alexander & & & & -1 \\
Menkeff & 8.02 & 2.34 & 2.35 & 11 \\
Jianan Wang & 8.18 & 2.33 & 2.22 & 16 \\
Xing-Long Li & 8.14 & 2.3 & 2.14 & -2 \\
Jin-zhe Li & 8.10 & 2.36 & 2.38 & 15 \\
Foreign Average & 8.22 & 2.47 & 2.32 & -12 \\
Domestic average & 8.14 & 2.33 & 2.45 & \\
\hline
\end{tabular}

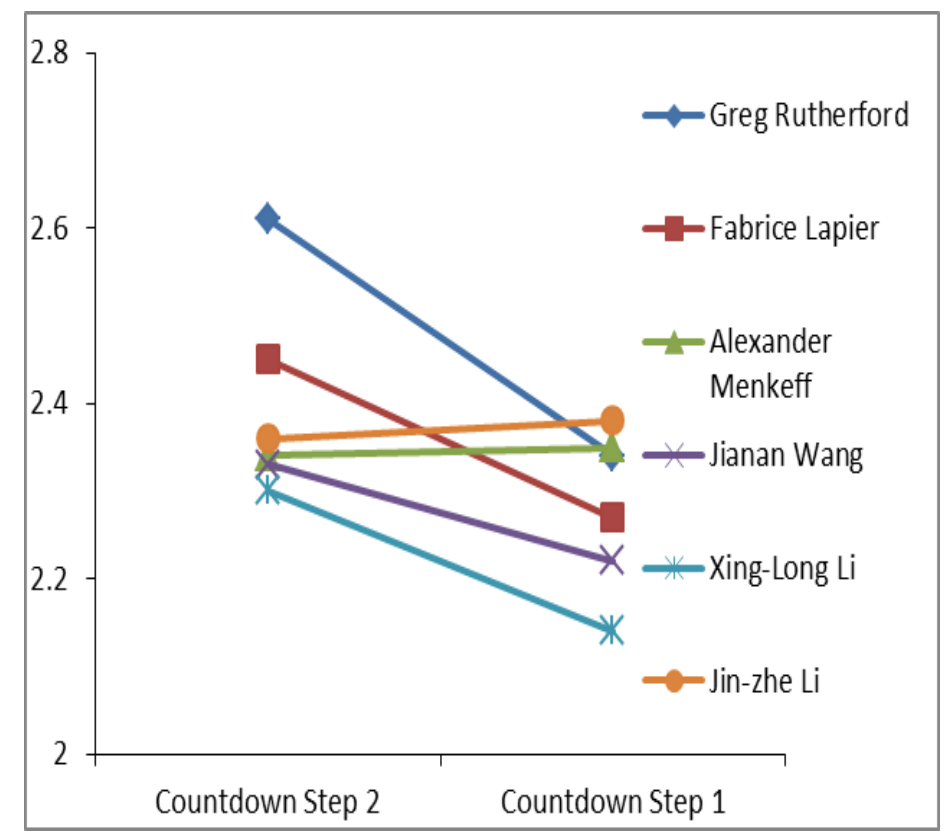

Fig. 1. Comparison of the last two steps

\section{B Takeoff Technique}

From the point of view of sports biomechanics, take-off is the turning point of the long jump from horizontal to ejection. And the appropriate angle of jump [5]

From the take-off stage, the quality of the body's jumping has a close relationship with the landing angle, the take-off sector angle and the angle of the take-off angle. Therefore, the angle change of the three different phases in the take-off stage directly reflects the athlete's body function state and movement technology Level [6] table III for the 2015 World Athletics Championships men's long jump event the first six athletes take-off phase of the take-off when the relevant parameters of the data. 
TABLE III. LONG Jump ATHLETES TAKE - OFF RELATED ANGLE

\begin{tabular}{ccccc}
\hline Name & $\begin{array}{c}\text { Achieve } \\
\text {-ment }\end{array}$ & $\begin{array}{c}\text { Landing } \\
\text { Angle }\end{array}$ & $\begin{array}{c}\text { Jump } \\
\text { Angle }\end{array}$ & $\begin{array}{c}\text { Sector } \\
\text { Angle }\end{array}$ \\
\hline $\begin{array}{c}\text { Greg } \\
\text { RUTHERFORD } \\
\text { Fabrice }\end{array}$ & 8.41 & 52.5 & 22.9 & 33.9 \\
$\begin{array}{c}\text { LAPIERRE } \\
\text { Aleksandr }\end{array}$ & 8.24 & 47.6 & 21.4 & 38 \\
MENKOV & 8.02 & 46 & 20.5 & 31.7 \\
Jianan Wang & 8.18 & 56.2 & 21.2 & 34.4 \\
Xinglong GAO & 8.14 & 44.3 & 20.9 & 47.6 \\
$\begin{array}{c}\text { Jinzhe Li } \\
\text { Foreign Average }\end{array}$ & 8.10 & 45.5 & 21.6 & 56 \\
$\begin{array}{c}\text { Domestic } \\
\text { Average }\end{array}$ & 8.22 & 48.7 & 21.6 & 34.5 \\
\hline
\end{tabular}

Take-off angle refers to the jump from the foot to the ground moment, the starting point and the body center of gravity line and the angle formed between the horizontal planes [3], take-off angle in relation to the rapid movement can use horizontal speed to obtain a larger vertical speed.

From table III we can see that the average angle of the domestic and foreign athletes in China is $48.6^{\circ}$ and $48.7^{\circ}$, and the difference is not significant between the two countries, and Xiaojin Feng's athletes who have scored more than 8 meters in China have found that the average angle and the value of $58.65^{\circ}$ [7], among the six long jump athletes, only Jianan Wang closest to $56.2^{\circ}$.

Jump angle is the end of take-off, from the ground to take off; the direction of the center of gravity displacement and the angle of the ground, the angle of the jump off the long jump play an important role in the decision [7]. After the jump, the horizontal speed will be decomposed into the level of speed and take off the vertical speed Jump, a reasonable point of Jump angle to minimize the loss of the horizontal speed, access to the level of speed is very small, The larger the vertical speed of the pitch, increase the displacement distance [8]

Table III shows that Greg RUTHERFORD of the six athletes has the maximum angle of $22.9^{\circ}$, the mean value of Chinese athletes' jumping angle is $21.2^{\circ}$, while the average value of foreign athletes is slightly larger than that of $21.6^{\circ}$ in China, The relevant research shows that $18^{\circ}-24^{\circ}$ is more appropriate Jumping starting point [8, 9], can be seen, our excellent athletes Jumping angle and foreign athletes are in a reasonable range, but still need to improve.

Take-off fan angle (sector angle) refers to the moment board with the body center of gravity of the connection and take-off end of the moment with the plate center of gravity and the angle of the connection line. The size of the sector angle indicates the distance the body center of gravity moves forward and the speed of the athlete's takeoff during the take-off [10]. A bigger sector angle indicates a higher vacating height.

As the training conditions of different athletes, fan size and specific location of the angle will be different, the success of the take-off technology on the board in the less timeconsuming, take-off faster, better performance, but also the key from the active take- Reduce the ground to the buffer braking force, so that the body center of gravity forward, timely fast pedal stretch to complete the take-off technical action, The jump speed of the high-jump athlete's fan-angle is small, but the vertical speed is high. The low-jump athlete has large fanangle and low horizontal velocity loss, and the vertical velocity is small. According to the table data, the jump angle of the foreign athletes is $34.5^{\circ}$, and the angle of the take-off fan of Chinese athletes is slightly larger than that of $46^{\circ}$. The six athletes in our study, Jinzhe $\mathrm{Li}$ and Xinglong Gao, The required take-off fan angle is $28^{\circ}-43^{\circ}$ [5] compared to slightly larger, indicating that our athletes are low jump athletes. It can be seen that our athletes are low-jump athletes with a small starting angle and a large fan angle, which will affect the coherence of running and jumping. It is not propitious to obtain the vertical speed, so we should take active and effective measures to improve the landing angle and take-off sector Angle, to strengthen the buffer capacity of the training, so that it reaches a reasonable range.

From the above analysis, it can be concluded that the angle of take-off of Chinese male long-jump athletes is small compared with that of foreign athletes: the angle of landing is small, the angle of tan is small and the angle of fan is big; compared with the reasonable range proposed by theoretically: The small angle of rise, and the larger fan angle, so that the cohesion of run-up take-off reduced, can't use better horizontal speed to get a larger take-off vertical speed. Therefore, for our male long-jump athletes, in future training should continue to strengthen the strength training and pedal extension training, to improve the angle, angle and takeoff jump angle fan angle, increasing the displacement of the purpose.

\section{CONCLUSIONS AND SUGGESTIONS}

In the last two steps of the run-up study, the second step of the last step of the foreign athletes is bigger than the $14 \mathrm{~cm}$ of our country, and the last step of the last step is bigger than that of the foreign athletes, especially the second step of Jinzhe Li is slightly smaller than the last step. It is suggested to reduce the last step and increase the training to reduce the loss of the last few steps of the run-up and prevent the decrease of the center of gravity of the approach and take-off stage.

The average angle of take-off angle of Chinese athletes is not very different from that of foreign athletes. Except for JiaNan Wang and Rutherford-Greg, the six athletes have a similar starting angle of $58.65^{\circ}$ and the other athletes are smaller. Therefore, in the training process, Attention to strengthen the body center of gravity rapid forward, fast, smooth on the plate, combined with their characteristics appropriate increase the angle of take-off angle to take full advantage of the horizontal speed to get a better take-off vertical speed.

Chinese athletes take-off jump angle and foreign athletes have little difference, and are in a reasonable range of values, but still need to strengthen training, appropriate to raise the angle, the use of vertical speed, the maximum displacement distance.

Chinese athletes, Jinzhe Li and Xinglong Gao belong to the low jump type athletes, the jumping fan angle is big; the horizontal speed loss is small, the vertical speed is small, it is recommended to strengthen the take-off pedal technology exercises, speed up the buffer after the pedal action, pay 
attention to maintaining the correctness and stability of technical training to improve the level of pedal moment speed and the appropriate angle.

\section{ACKNOWLEDGMENT}

This work was supported by the Open Project Program of Key Lab of Training, Monitoring and Intervention of Aquatic Sports of General Administration of Sport of China, Jiangxi Normal University (NO. 201604).

\section{REFERENCES}

[1] H.G. Yan, T.F.LV, "Kinematics Analysis of the Last Two Steps and Take - off Technique of Chinese Elite Male long jumpers”, Liaoning Sport Science and Technology, PP.33-34, 1994.(In Chinese)

[2] F.Y.Liu, "Kinematical Analysis on the Last Two Steps of Approach and Take - off Technique of Male long jump Athletes in Some Colleges and Universities”

[3] J.Zhang, M.X.LU, "Kinematics Analysis on the Last Two Steps of Chinese Elite Female Long Jumper”, Hebei Physical Education Institute, Vol.06, PP.84-86+96, 2009. (In Chinese)
[4] Y.G. Yuan, A.D. Li, F.Q. Zheng, Z.F. Wang, Y.X. Mi, and G.J. Wang, "The 2014 Beijing international athletics challenge men's long jump athletes Jin-zhe Li key movement technology research” ,Chinese sports science and technology , 6th ed.Vol.50 , PP.29-35, 2015 .(In Chinese)

[5] Q. Li, "Excellent male long jump athletes in our country after four steps approach and take-off technology analysis of the kinematics” ,Hebei normal university , 2009.(In Chinese)

[6] L.K. Zhang, W. Zhang, X.F.L, "Research on Angle Parameters of Long Jump” ,Harbin Institute of Physical Education, Vol.04, PP.110-112, 2002.(In Chinese)

[7] X.J.Feng, "From the Take - off Link to See the Gap Between the Domestic and Foreign Player 's long jump” ,Shanghai Institute of Physical Education, Vol.01, PP.60-62, 1999.(In Chinese)

[8] J.S. Shan, Q.J. Ren, C.H. Li, G.Q. Feng, “Analysis and Research on Jumping Angle of Men 's long jump” ,Juvenile sports training , Vol.01, PP.43, 2011.(In Chinese)

[9] James G. Hay, John A. Miller, Ron W. Cantner and X.F. Wang, "Biomechanical Analysis of Elite long jumpers" ,Journal of Shandong Sports Science, Vol.03, PP.32-35, 1998.(In Chinese)

[10] Q.J. Pang, "Kinematical Analysis on the Last Two Steps and Take - off Phase of the Outstanding Male long jumpers" ,Wuhan Institute of Physical Education, 2006.(In Chinese) 